\title{
Turbulence and dispersion below and above the interface of the internal and the external boundary layers
}

\author{
Vincenzo Sessa $^{\mathrm{a}}$, Zheng-Tong Xie ${ }^{\mathrm{a}, *}$, Steven Herring ${ }^{\mathrm{b}}$ \\ ${ }^{a}$ University of Southampton, Southampton, SO17 1BJ, UK \\ ${ }^{b}$ Dstl, Porton Down, Salisbury, SP\& OJQ, UK
}

\begin{abstract}
This study has looked at the development of the internal boundary layer (IBL) over a block array close to a sharp change in surface roughness and its effect on dispersion from a ground level source for ratios of the downstream distance to the roughness length of less than 300 . This was done by comparing a Large-Eddy Simulation (LES) with inflow boundary conditions against a LES with inlet-outlet periodic boundary conditions and data from a wind tunnel experiment. In addition to established methods, an alternative approach based on the vertical Reynolds stress was used to evaluate the depth of the IBL as it developed over the array which enabled the location of the interface to be more clearly defined. It was confirmed that the IBL growth rate close to the change in surface roughness could be described by a power law profile, similar to the power law formula used in previous studies for a ratio of the downstream distance to the roughness length greater than 1000. An analysis of mean concentration and turbulent scalar fluxes suggested that the presence of the IBL constrained the vertical development of the plume from a ground level source and so led to trapping of material in the canopy layer.
\end{abstract}

Keywords: IBL, inflow turbulence, dispersion, urban environments

\footnotetext{
${ }^{*}$ Corresponding author

Email address: Z.Xie@soton.ac.uk (Zheng-Tong Xie)
} 


\section{Introduction}

2 Urban roughness is heteogenous consisting of different type of surfaces (e.g. 3 Antoniou et al., 2016, King et al., 2017, Vasaturo et al., 2018, Tolias et al., 4 2018). A change in surface roughness, such as exists at the edge of the city, ${ }_{5}$ or at the junction between the central business district (CBD) and an area of 6 surrounding low-rise buildings, leads to a region of transitional flow as the tur-

7 bulent boundary layer takes time to adapt to the new wall condition (e.g. Smits 8 \& Wood 1985, Cheng \& Castro, 2002, Barlow, 2014, Cao \& Tamura, 2007, 9 Hanson \& Ganapathisubramani, 2016). This transitional flow results from the 10 development of an internal boundary layer (IBL) above the roughness elements. ${ }_{11}$ It is challenging to identify the interface of the IBL and the approaching bound12 ary layer. The interface may have a crucial effect on scalar exchange. To gain a deeper understanding of dispersion from a ground level source downstream of a change in surface roughness, it is important to analyse the development of the IBL.

Barlow (2014) analysed a turbulent boundary layer approaching a rural-tourban transition region (rough-to-very rough surface change) and found that on the city scale an IBL began to form at the junction between the smoother rural and the rougher urban surfaces. However, on the neighbourhood scale, and close to the ground, the flow was continuously adjusting to changes in roughness and that locally generated IBLs were in equilibrium with the underlying surfaces. This meant that multiple changes in roughness could lead to overlapping local IBLs and, as a consequence, the IBL assumed a non-homogeneous 3 -dimensional structure extending up to $2-5$ times the mean building height 25 Barlow \& Coceal, 2008). These findings were supported by those of Hanson \&

26 Ganapathisubramani (2016), who analysed boundary layer development across ${ }_{27}$ a rougher-to-smoother surface change and identified an IBL with two regions: ${ }_{28}$ an energetic region near the wall in which the flow had adapted to the new wall condition and an outer region in which the flow retained characteristics of the so upstream condition. 
In order to investigate the effects of the IBL it is first necessary to define its outer limit. Some authors (e.g. Cheng \& Castro, 2002; Schlichting \& Kestin 1979) have defined the height of the IBL as the point on a log-linear plot where the velocity reaches $99 \%$ of that for upstream roughness at the same position. However, this definition is difficult to use because of the uncertainty of measuring differences of $1 \%$ experimentally or numerically. An alternative method (Antonia \& Luxton, 1972) involves plotting the streamwise velocity profile at a series of locations against the square-root of the height above ground. The profiles are then approximated by two straight line segments which correspond to the external and IBLs, and the intersection of the segments defines the height of the IBL. The method devised by Efros \& Krogstad (2011) is similar, but involves plotting the streamwise Reynolds stress against the height. The depth of the IBL is again indicated by the intersection of two lines. Both of these methods are based on the "knee" point technique which might be open to interpretation in situations where the differences between the boundary layer velocity profiles are small. Nevertheless, they are easy to implement and provide a good indication of the IBL growth rate.

The strength of the step change in roughness between two regions may be described by the roughness length ratio $\left(z_{01} / z_{02}\right)$, where $z_{01}$ and $z_{02}$ are the upstream and downstream roughness lengths respectively. Despite this, Jackson (1976) used atmospheric and wind-tunnel data to demonstrate that the growth rate of the IBL is essentially driven by the rougher surface and is not related to the roughness length ratio, or to the ratio of boundary-layer thickness to roughness element height. This conclusion is supported by work conducted by Townsend (1965) and Schofield (1975), which showed that if $\left(z_{01} / z_{02}\right)<1$ (a change from smoother to a rougher wall) only $z_{02}$ is important in estimating the growth of the IBL.

Bradley (1968) conducted the first atmospheric experiments on IBL development due to an increase in surface roughness. His observations showed that the growth rate of the IBL was independent of wind speed and described well by Elliot's formula (Elliott, 1958) which also indicates that the IBL development 
is independent of the roughness length ratio:

$$
\frac{\delta}{z_{02}}=a\left(\frac{X}{z_{02}}\right)^{P}
$$

where $X$ is the distance from the step change, $\delta$ is the depth of the IBL, and $P$ and $a$ are the exponent and the coefficient of the power-law formula respectively. Elliott (1958) found that the coefficient $a$ varied from 0.6 to 0.9 and that $\mathrm{P}=0.8$ for $X / z_{02}>1000$. His work has been further confirmed by (Wood, 1982 , Pendergrass \& Arya, 1984). Elliot emphasised that the exponent $P=0.8$ in Eq. 1 was only valid for $X / z_{02} \geq 1000$ as it relied upon assumptions of constant stress and a logarithmic profile that were only valid downstream of that point. He showed that closer to the roughness transition point the development of the IBL could be fitted with a similar power law relationship, but with a lower exponent $P$ and greater coefficient $a$.

Cheng \& Castro (2002) performed an experimental study of the flow field immediately downstream of a roughness transition in which they fitted their experimental results for the IBL at distances of $X / z_{02}<1000$ by applying Eq. 1 using an exponent $P=0.33$ and a coefficient $a=10.56$. This confirmed Elliott's finding that the exponent $P$ decreased while the coefficient $a$ increased as $X / z_{02}$ was reduced. In their work Cheng \& Castro (2002) defined the IBL height as the location where the velocity was $99 \%$ of that for upstream roughness at the same height. This led to significant scatter in the derived IBL height due to the difficulty of measuring $1 \%$ differences experimentally.

Large-Eddy Simulations have demonstrated the capability to model turbulence, dispersion and heat transfer in urban environments (Baker et al., 2004. Fuka et al., 2017, Hanna et al., 2002, Kanda et al., 2004, Xie \& Castro, 2006). The majority of LES flow and dispersion studies have focussed on the streetscale below and immediately above the canopy and used simple periodic inletoutlet boundary conditions. Very few numerical studies have analysed the characteristics of turbulent flow as it passes over a change in roughness. Two which have are those by Michioka et al. (2011) and Tomas et al. (2017). Both of these applied the LES approach using the inflow boundary condition method to com- 
pute the flow over arrays of obstacles with various aspect ratios with the aim of better understanding the dispersion mechanisms. However, their focus was limited to examination of the advective and turbulent pollutant fluxes around the array obstacles up to the canopy height, and they did not examine IBL development. To the authors' knowledge there have been no numerical studies or experimental works which have specifically addressed the effects of IBL development on gas dispersion.

In this paper we examine LES predictions made using the inflow generator proposed by Xie \& Castro (2008), and then identify how the height of the IBL evolves over a psuedo rural-to-urban transition region using a similar approach to those proposed by Efros \& Krogstad (2011) and Antonia \& Luxton (1972), but based on using the vertical Reynolds stress. This was done with the objective of understanding the extent to which the dispersion of a neutrally buoyant gas from a ground-level point source is influenced by the interaction of the external boundary layer and IBL. The governing equations are briefly described in Sect. 2 Details of the numerical settings including geometry, mesh and inflow conditions are given in Sect. 3 LES predictions for turbulence and dispersion are discussed in Sect. 4. Finally, the conclusions are summarised in Sect. 5

\section{Governing equations}

In LES the filtered continuity and Navier-Stokes equations are written as follows:

$$
\frac{\partial \bar{u}_{i}}{\partial x_{i}}=0
$$

$$
\frac{\partial \bar{u}_{i}}{\partial t}+\frac{\partial \bar{u}_{i} \bar{u}_{j}}{\partial x_{j}}=-\frac{1}{\rho} \frac{\partial \bar{p}}{\partial x_{i}}+\frac{\partial}{\partial x_{j}}\left(\frac{\tau_{i j}}{\rho}+\nu \frac{\partial \bar{u}_{i}}{\partial x_{j}}\right)
$$

where the filtered velocity and pressure fields are $\bar{u}_{i}$ and $\bar{p}$ respectively, $\nu$ is the kinematic molecular viscosity and $\rho$ is the density. The standard Smagorinsky subgrid-scale (SGS) model was applied to determine the isotropic part of the residual stress tensor $\tau_{i j}$ :

$$
\tau_{i j}=-2 \nu_{r} \bar{S}_{i j}
$$


where $\bar{S}_{i j}=\frac{1}{2}\left(\frac{\partial \bar{u}_{i}}{\partial x_{j}}+\frac{\partial \bar{u}_{j}}{\partial x_{i}}\right)$ is the filtered rate of strain and $\nu_{r}$ is the SGS residual viscosity. The SGS residual viscosity $\nu_{r}$ is modelled as:

$$
\nu_{r}=\left(C_{S} \Delta\right)^{2} \bar{S}
$$

where $\bar{S}$ is the characteristic filtered rate of strain, the filter width $\Delta$ was taken as the cube root of the cell volume and the Smagorinsky coefficient as $C_{S}=0.1$. The Van Driest damping function was applied in the near wall region.

The Smagorinsky model supplemented with a wall-damping function is known to be less accurate near a flow-reattachment point or in free-shear layer regions (Inagaki et al. 2005). However, Castro et al. (2017) compared the performance of the dynamic mixed time scale sub-grid model (Inagaki et al. 2005) and the standard Smagorinsky over an identical array of uniform blocks and their results revealed only small differences in the spatially averaged mean velocities and turbulence stresses. Based on those results the standard model and wall-damping function were adopted for the simulations reported here.

The filtered transport equation for a passive scalar is:

$$
\frac{\partial \bar{C}}{\partial t}+\frac{\partial \bar{u}_{j} \bar{C}}{\partial x_{j}}=\frac{\partial}{\partial x_{j}}\left[\left(K+K_{r}\right) \frac{\partial \bar{C}}{\partial x_{j}}\right]+S
$$

where $\bar{C}$ is the filtered scalar concentration and $S$ is a source term. The second term on the left-hand side is the advection term and the first term on the righthand side is the diffusion term. $K$ is the molecular diffusivity and $K_{r}$ is the SGS turbulent diffusivity computed as:

$$
K_{r}=\frac{\nu_{r}}{S c_{r}}
$$

where $S c_{r}$ is the subgrid Schmidt number. A constant Schmidt number of $S c_{r}=0.7$ was assumed.

\section{Numerical settings}

The LES model was implemented within the open-source CFD package OpenFOAM version 1.7.1. A second-order backward implicit scheme in time and 
second-order central difference scheme in space were applied for the discretisation of the terms in Eqs. 3 and 6 . Flow and turbulence within the domain were simulated as a half channel. An efficient inflow turbulence generation method (Xie \& Castro, 2008) was used at the inlet, with periodic conditions at the lateral boundaries and a stress-free condition at the top of the domain ( $y=12 h$, where $h=70 \mathrm{~mm}$ is the uniform height of the array element). The Reynolds number based on $h$ and the free stream velocity $U_{\text {ref }}=2 \mathrm{~m} / \mathrm{s}$ at $y=12 h$ was approximately 12,000. The averaged CFL (Courant-Friedrichs-Lewy) number was 0.2 , based on a time step resolution of $0.0014 \mathrm{~s}$. Flow and second-order statistics were initialized for $40 \mathrm{~s}$ and then averaged over $180 \mathrm{~s}$ (180 flow-passes).

\subsection{Geometry, mesh and resolution}

Although computational power is increasing, the simulation of most real urban scenarios still represents a challenge, especially when large and complex geometries have to be analysed. In studies to improve the understanding of building aerodynamics it is therefore usual to represent urban configurations in their simplest form as an array of cuboids in regular or non-regular patterns (e.g. Hanna et al. 2002, Xie \& Castro, 2006).

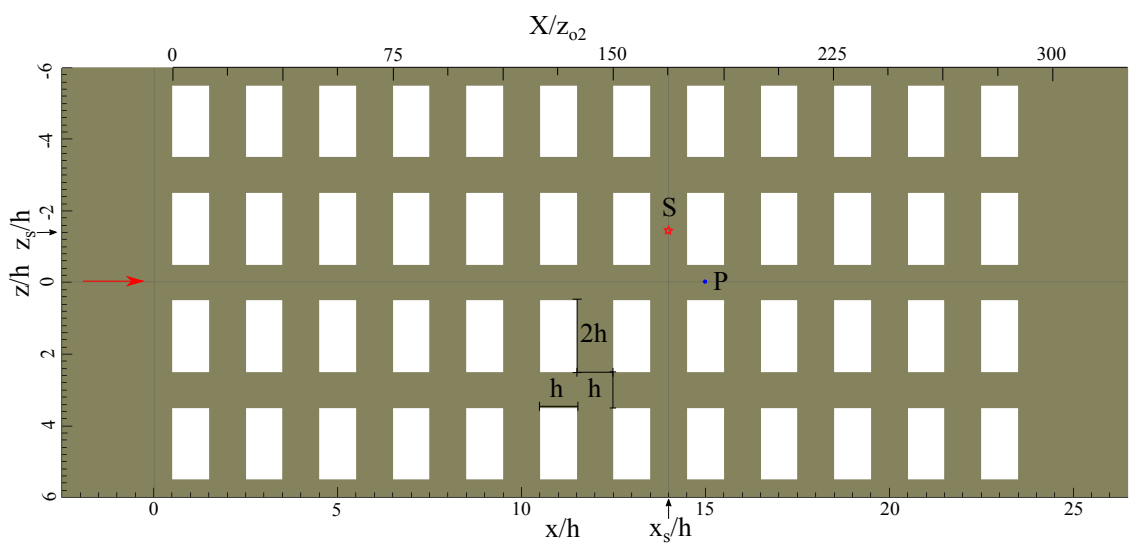

Figure 1: The array configuration: dimensions of buildings and streets, the coordinate system, the flow direction, the distance from the leading edge $X / z_{02}$, the position $x_{s}$ and $z_{s}$ of source $S$ and measurements location P. 
The array of regular cuboids modelled in this paper represents part of a larger array used in a wind tunnel experiment designed to simulate a neighbourhood scale region in which statistical homogeneities should exist. On the basis that the length of the streets should exceed the building height $h$, to establish developed street-canyons flows, Castro et al. (2017) adopted cuboid obstacles with dimensions $1 h($ length $) \times 1 h($ height $) \times 2 h($ width $)$. The section of the array modelled is shown in Fig. 1 where the street units parallel to the $x$ axis are $1 h$ long and referred to as 'short streets' hereinafter. Street units parallel to the $z$ axis are $2 h$ long and referred to as 'long streets'. The rectangular array comprised 48 aligned blocks with $h$ spacing, which considering the single block unit leads to a plan area density of $\lambda_{p}=0.33$.

The dimensions of the modelled domain were $29 h \times 12 h \times 12 h$ within a uniform Cartesian grid of resolution $\triangle=h / 16$. Simulations by Castro et al. (2017) at this resolution yielded LES results for turbulence that agreed well with higher resolution Direct Numerical Simulation (DNS) data. The top boundary was placed at $y=12$, which is very close to the boundary layer height in the wind tunnel. Castro et al. (2017) performed a sensitivity test of smaller domain heights $(y=6 h, 8 h$ or $10 h)$ and recommended a top domain of at least six canopy heights in order to capture the most important turbulence features. Computations were made for the $0^{\circ}$ wind direction by assuming that the mean wind flow was perpendicular to the front face of the cuboid elements as indicated in Fig. 1 .

\subsection{Scalar source}

A passive scalar was released from a ground-level point source within the array of cuboid elements. The shape and size of the point source were identical to that reported in Fuka et al. (2017). The source was positioned at the middle of a long street after the seventh row of buildings (Fig. 1) where the downstream flow would be fully developed (Hanna et al. 2002). Because the modelling used a uniform grid, the shape of the source only approximated the source used in the experiment. The diameter was represented by 4 cells and so measured $0.25 h$, 
while the height was one cell $(h / 16)$. A constant scalar flux release rate was set for each cell inside the source volume.

\subsection{Inflow conditions}

The simulation of flow over a rural-to-urban transition region requires a continuous specification of inlet turbulence. This was achieved by using the inflow turbulence method developed by Xie \& Castro (2008) to generate a synthetic turbulent inflow with exponential-form correlations in time and space. The used inflow method proved to reconstruct energy-containing region and inertial sublayer of the spectra in high fidelity. Moreover, (Bercin et al., 2018) showed that the use of exponential-form correlation functions as a model approximation is more advisable than that of Gaussian-form.

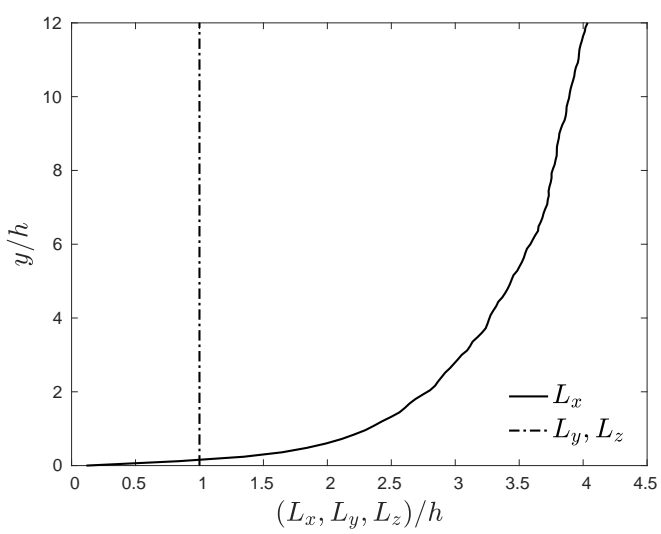

Figure 2: Vertical profiles of prescribed integral length scales at the LES inlet $x=-2.5 h$. 


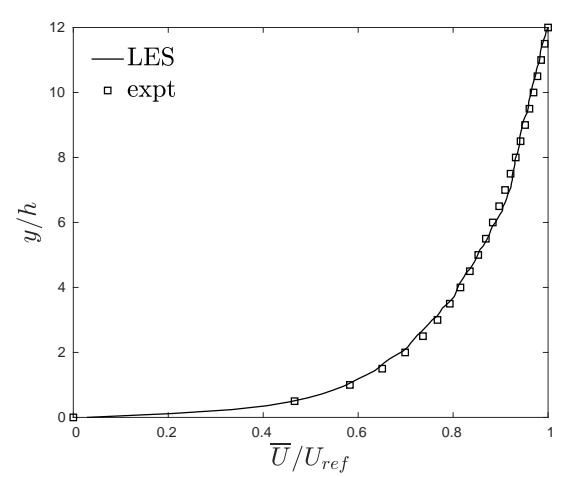

(a)

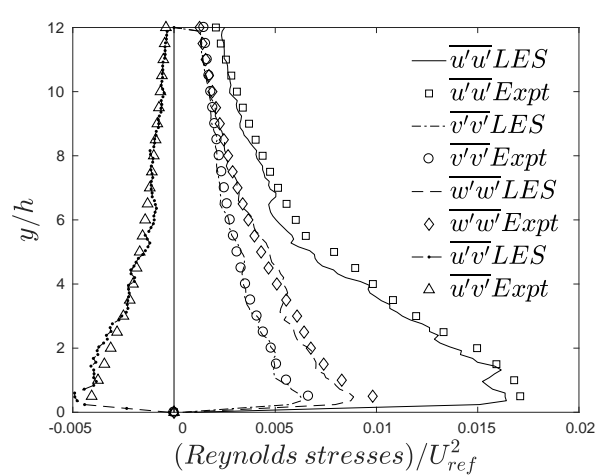

(b)

Figure 3: (a) Vertical profiles of laterally averaged mean velocity from LES at inlet and prescribed mean velocity from experiments. (b) Vertical profiles of prescribed Reynolds stresses at the LES inlet and experimental values.

The generated turbulence satisfied the prescribed integral length scales and Reynolds stress-tensor values. The integral length scales $L_{x}, L_{y}$ and $L_{z}$ in the streamwise, vertical and lateral directions respectively were estimated from data presented in Castro et al. (2006) and shown in Fig. 2. Xie \& Castro (2008) performed LES for different length scale combinations imposed at inlet (i.e. $L_{x}, L_{y}$ and $L_{z}$ factored by $0.5,1$ or 2 ). It was found that the results of mean velocity and turbulent stresses within or immediately above the canopy were insensitive to the precise inflow length scales.

The prescribed mean velocity and Reynolds stresses were obtained from the wind tunnel experiment reported in Castro et al. (2017) by assuming lateral homogeneity. Fig. 3a shows the prescribed mean velocity profile. Castro et al. (2017) fitted the profile in the usual $\log -\operatorname{law}$ form $U=\frac{u^{*}}{\kappa} \ln \left(\frac{z-d}{z_{0}}\right)$ with $z_{01}=1.8 \mathrm{~mm}, d=0$ and by assuming $\kappa=0.41$. They also estimated that the friction velocity $u^{*}$ is $0.067 U_{\text {ref }}$ at $7 \mathrm{~h}$ upstream of the array. This is consistent with the peak Reynolds shear stress measured at the same location. 


\section{Results}

The turbulence and dispersion predictions produced using the inflow method were compared against LES predictions made using periodic boundary conditions at the inlet and outlet and the wind tunnel experiment data reported in Castro et al. (2017). The Reynolds numbers of the flows over the array were similar in all three cases.

A representative atmospheric boundary layer profile was generated in the experiment by a set of Irwin spires at the entry of the working section and an array of thin $2 \mathrm{D}$ plates (height $\sim 0.3 h$ ) placed upstream of the cuboid array. As mentioned, the experimental and LES value of the upstream roughness length was $z_{01}=1.8 \mathrm{~mm}$ whereas the roughness length of the array of cuboid elements was $z_{02}=5.6 \mathrm{~mm}$ as in Castro et al. (2017). This meant that an IBL was created from the leading edge of the array which developed in the downstream direction.

The development of the IBL was captured by the simulation made using the turbulence inflow generation method with prescribed turbulence statistics $(\S 3.3$, but was not by the simulation based on using periodic boundary conditions (PBC) at the inlet and outlet. This was because the PBC simulation effectively modelled the array as a single repeated unit of an infinite domain. One might therefore expect the inflow boundary condition (IBC) simulation to give a more accurate prediction of flow characteristics measured in the experiment, not only within the IBL but also above it when compared to the PBC simulations.

\subsection{Flow and turbulence}

For a simulation to accurately predict the dispersion of a pollutant it must accurately predict the turbulence statistics of the flow. This was assessed by examining the mean velocity and second-order statistics in the middle of the short streets (e.g. position $P$ of Fig. 1) normalized by the reference velocity.

For the IBC case the results were averaged at the four locations equivalent to point $P$ in Fig. 1 after the seventh row of cuboids at $x=15 h$. Whereas the 
PBC results produced by Castro et al. (2017) were averaged over all equivalent locations within the array. The experimental data were averaged in time over 3 minutes, but not in space. The wind tunnel errors were assumed to be $2 \%$ for $\bar{U}, 10 \%$ for $\overline{u^{\prime} u^{\prime}}$ and $5 \%$ for $\overline{v^{\prime} v^{\prime}}$ and $\overline{w^{\prime} w^{\prime}}$, respectively.

The IBC predictions for mean velocity (Fig. 4a) were found to be in good agreement with the experimental data below the canopy and up to $y=2 h$. Above that height, the IBC results slightly over-predicted while the PBC underpredicted the mean velocity.

Fig. $4 \mathrm{~b}$ shows that the streamwise Reynolds stress was predicted more accurately by the IBC simulation than the PBC. The peak stress occurred at the canopy height and was successfully captured by the IBC. The Reynolds stress profile above the canopy was also well predicted. The PBC simulation underpredicted the peak streamwise stress at the canopy height, and over-predicted the streamwise stress for $y / h>3$. 


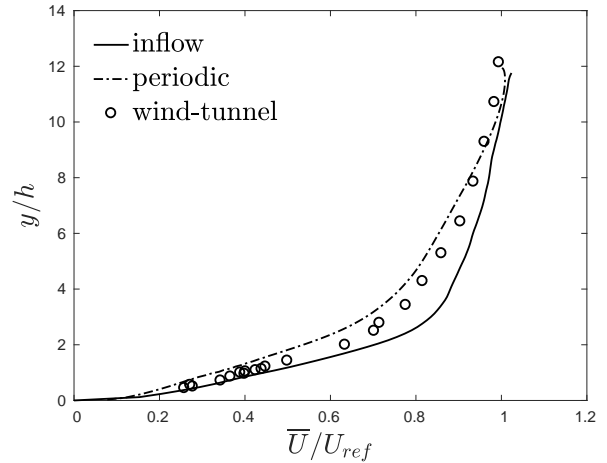

(a)

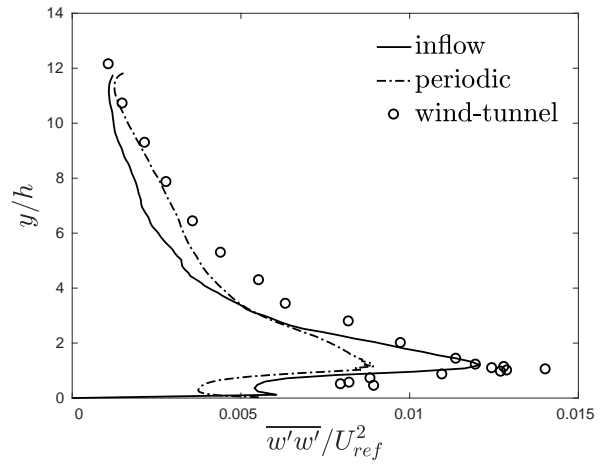

(c)

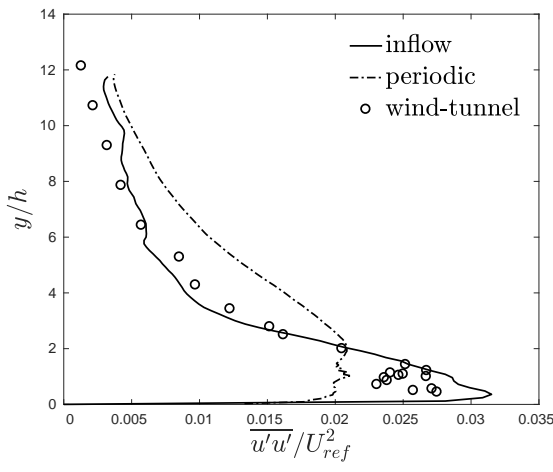

(b)

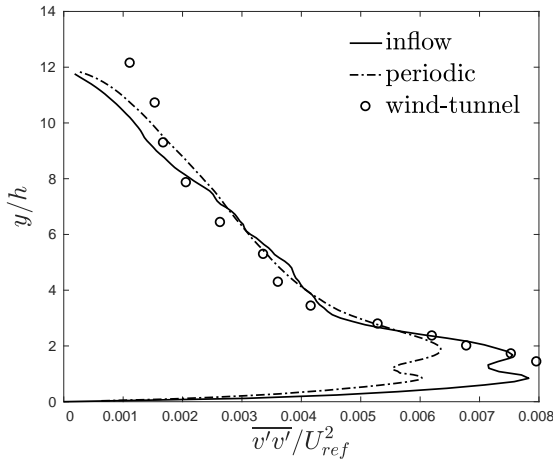

(d)

Figure 4: (a) IBC and wind tunnel mean velocity profiles measured at $x=15 h$ and spanwise averaged for position $\mathrm{P}$ in Fig. 1 Periodic mean velocity values were averaged at all similar points across the array. Corresponding profiles of streamwise normal stress (b), lateral normal stress (c) and vertical normal stress (d).

The lateral Reynolds stress, $\overline{w^{\prime} w^{\prime}}$, was well predicted by the IBC below and immediately above the canopy as shown in Fig. $4 \mathrm{c}$ but at greater heights it under-predicted the stress compared to the wind tunnel data.

The vertical stress (shown in Fig. 4d) was well predicted by both IBC and PBC simulations. Nevertheless, the inflow method gave more accurate results immediately above the canopy height. Close to the top of the domain, both LES computations underestimated the vertical stress because the vertical velocity gradient was fixed to zero by the symmetric boundary condition.

The results show that imposing inflow turbulence using IBC captured the 
transition from a rough to a very rough wall and led to predicted Reynolds stresses that were in better agreement with experimental results below and immediately above the canopy than those obtained from the PBC approach. This confirmed that the IBC method provided a better approach to predicting the characteristics of the flow below and immediately above the canopy.

\subsection{Growth of the internal boundary layer}

The transition from the relatively smooth surface ahead of the array to the much higher roughness of the array itself causes an IBL to develop from the leading edge of the obstacles. The IBL increases in depth as it develops downstream through the array and the flow within it is characterised by having greater turbulent kinetic energy (TKE) than that in the external boundary layer above it. As TKE is the primary driver of dispersion, it is expected that the location of the interface between the internal and external boundary layers will be have an important influence on the dispersion of material from a source within the array.

Three methods were used to determine the interface between the internal and external boundary layers along the length of the array in the IBC simulation. Method I was that developed by Antonia \& Luxton (1972). Fig. 5 shows the result of applying method I using normalized velocity profiles obtained by averaging over 48 lateral positions, for nine streamwise locations (a vertical offset is imposed for ease interpretation). The regions related to the internal and external boundary layers were then linearly fitted to a residual error of less than $2 \%$.

The first velocity profile was taken at $x=-2.5 h$ (3h upstream of the leading edge LE of the array in Fig. 1] where the mean velocity profile approaching the urban array is shown. There is no IBL at that point, and the profile cannot be linearly fitted. At the second location, at $x=2 h(1.5 h$ downstream of the leading edge) the profile is distinctly different and has two linear fits, whose intersection identifies the edge of the IBL $(x=2 h, y=1.8 h)$. Following the same approach, the edge of the IBL can be identified for a further 7 downstream 


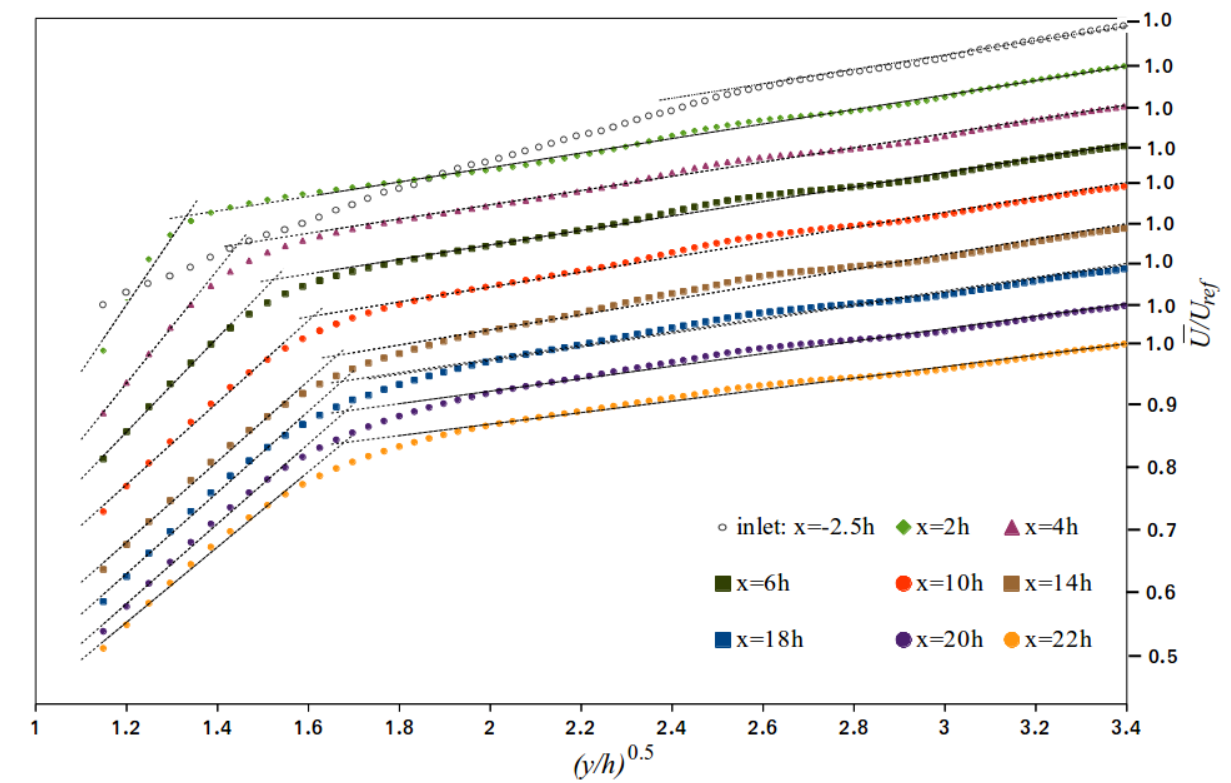

Figure 5: Laterally averaged mean velocity profiles in 9 streamwise locations: 1 upstream and 8 downstream of the leading edge (LE). The velocity profiles are shifted upwards to facilitate interpretation.

locations and the evolution of the interface derived.

Method II was that developed by Efros \& Krogstad (2011) based on plotting the streamwise Reynolds stress component normalized by the friction velocity $u^{*}$, i.e. ${\overline{u^{\prime} u^{\prime}}}^{+}$, against the height normalized by the domain height $H$. Method II was applied by calculating the streamwise stress profile at the same $x$-locations and averaged over 48 lateral positions as previously. The profiles within the external and IBL were then linearly fitted to a residual error less than $5 \%$. The edge of the IBL was again found at each location and its growth with downstream distance is shown in due course.

The flow over an array of cuboid obstacles is a complex, anisotropic 3D turbulent flow. This complexity means that if the TKE generated upstream of the leading edge is similar to that produced downstream, then identifying the interface between the internal and external boundary layers may be difficult.

A more accurate method for defining the edge of the IBL helps to 
analyse flow and dispersion mechanisms over such roughness transition regions, in particular in some specific scenarios such as in stable stratification (e.g. Kanda \& Yamao, 2016). A step change over cuboid elements with uniform height, perpendicular to the flow direction, determines a more visible interface. The interface is more well-defined as greater is the difference between the TKE below and above it. Therefore, a more accurate method for defining the edge of the IBL helps to analyse flow and dispersion mechanisms over such roughness transition regions.

Given that the IBL grows in the vertical direction and a laterally homogeneous flow is assumed above the canopy, then it may be hypothesised that the use of a wall-normal parameter may make identifying the interface easier.

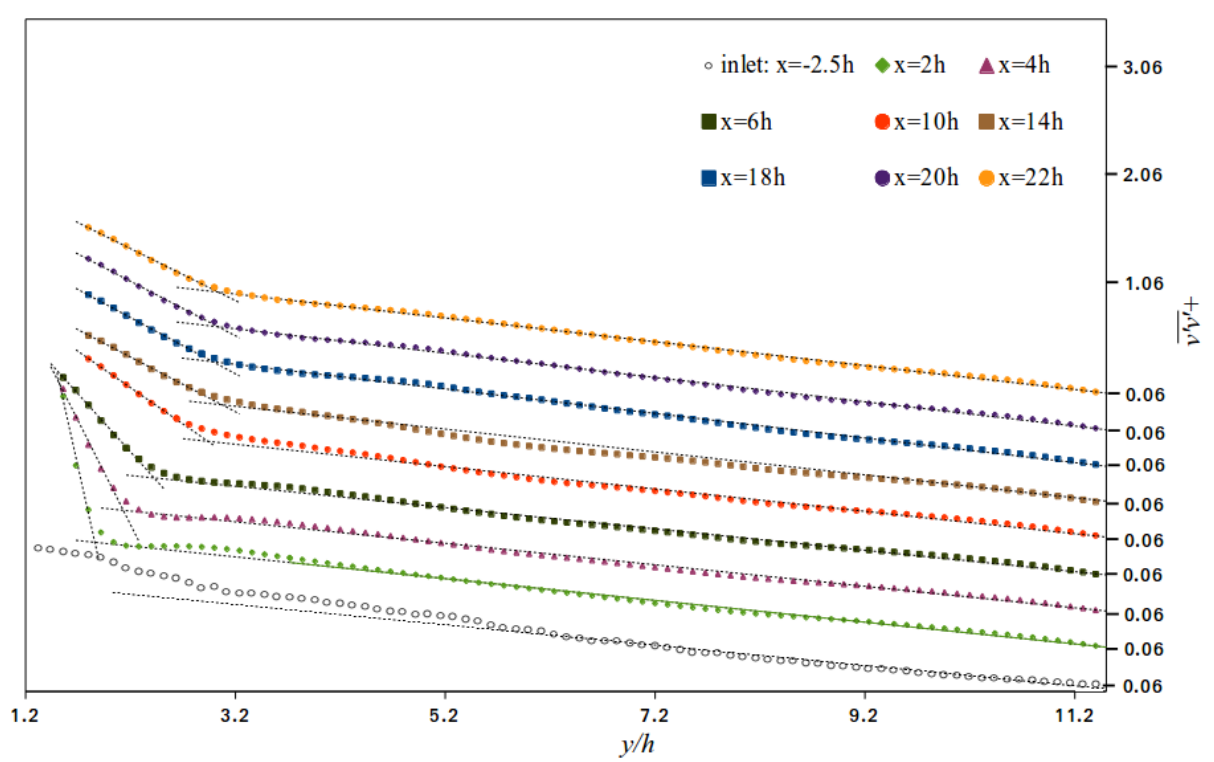

Figure 6: Laterally averaged vertical Reynolds stress profiles at 9 streamwise locations: 1 upstream and 8 downstream of the leading edge (LE). The stress profiles are shifted downwards to facilitate interpretation.

This paper tests a method based on the wall-normal turbulent variance $\overline{v^{\prime} v^{\prime}}$, referred to as method III. Fig. 6 shows the vertical Reynolds stress profiles 
normalized by the friction velocity $u^{*}$, i.e. $\overline{v^{\prime} v^{\prime}}+$, plotted against the height normalised by the domain height $H$, in a similar way to applying methods I and II. The Reynolds stress profiles for the external and IBL regions were linearly fitted to a residual error of less than $1 \%$.

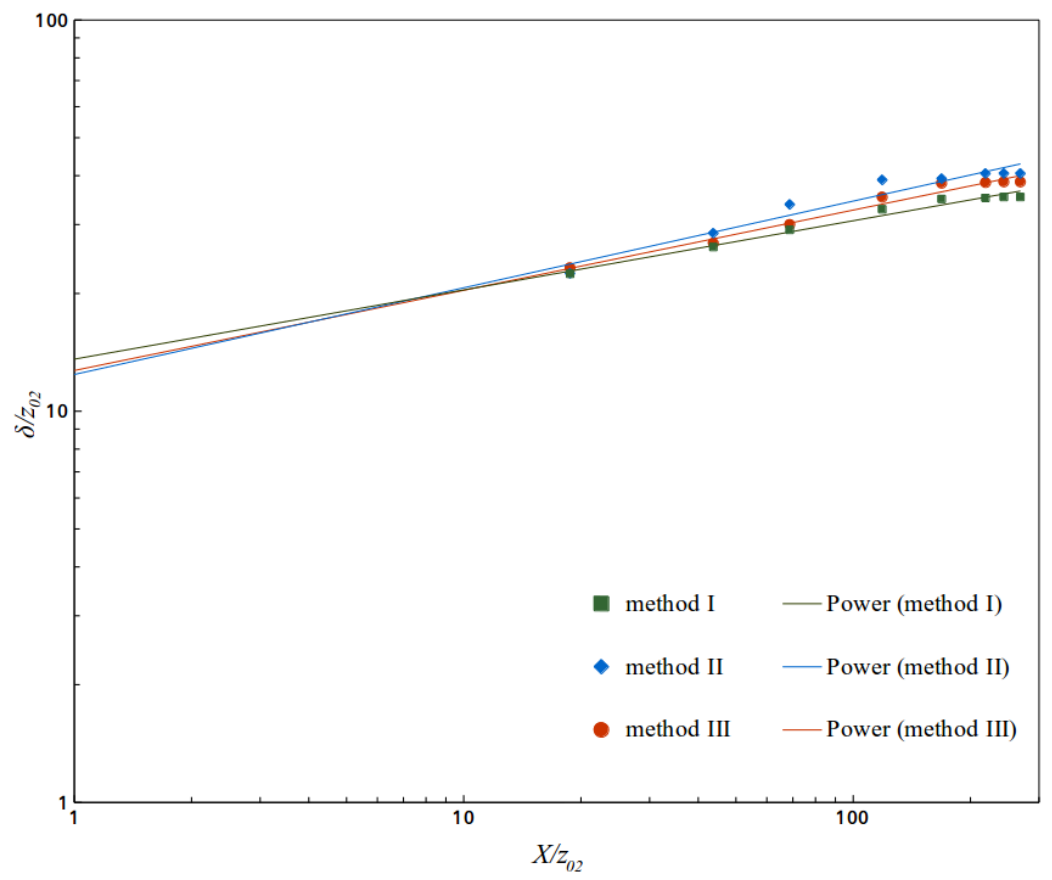

Figure 7: IBL depth $\delta$ derived by using the mean velocity $\bar{U}$ (method I, square green), the streamwise stress $\overline{u^{\prime} u^{\prime}}$ (method II, diamond blue) and the vertical stress $\overline{v^{\prime} v^{\prime}}$ (method III, circle red). These data fit to power-law profiles with lines respectively.

Fig. 7 shows the result of fitting the IBL depth data derived from the three methods to the Elliott (1958) power-law formula (Eq. 1). The residual error of the power-law fit for method I $(\bar{U})$ was less than $3 \%$ with exponent $P=0.18$ and coefficient $a=13.59$ :

$$
\frac{\delta}{z_{02}}=13.59\left(\frac{X}{z_{02}}\right)^{0.18}
$$

The residual error of the power-law fit for method II $\left(\overline{u^{\prime} u^{\prime}}\right)$ was less than 
$6 \%$ with exponent $P=0.22$ and coefficient $a=12.42$ :

$$
\frac{\delta}{z_{02}}=12.42\left(\frac{X}{z_{02}}\right)^{0.22}
$$

Lastly, the residual error of the power-law fit for method III $\left(\overline{v^{\prime} v^{\prime}}\right)$ was less than $2.5 \%$ with exponent $P=0.21$ and coefficient $a=12.71$ :

$$
\frac{\delta}{z_{02}}=12.71\left(\frac{X}{z_{02}}\right)^{0.21} .
$$

The fitted results (Eqs 810 from the three methods all confirmed that near the step change in roughness $\left(X / z_{02}<300\right)$ the exponent $P$ of the power-law formula is much lower than the value $P=0.8$ found in literature for $X / z_{02}>$ 1000. Moreover, the estimated coefficient $a$ is much higher than the range of values suggested by Elliott (1958). This is perhaps not surprising as the fitted results are dependent on the details of step change in roughness and the characteristics of the roughness elements.

The fitted results from the three methods are consistent. Of the three methods analysed here, the IBL heights derived from method III were fitted to the power-law formula with the lowest residual error. The coefficient $a=12.71$ is not significantly different from $a=10.56$ obtained in Cheng \& Castro (2002), which studied the height of an internal boundary layer over an array of twodimensional rib-type roughness elements at a range $X / z_{02}<1000$. Nevertheless, the exponent $P=0.21$ was significantly different from that $P=0.33$ found by Cheng \& Castro (2002). Again, this is owing to the difference of the roughness elements between the current study and Cheng \& Castro (2002). We speculate that the two-dimensional rib-type elements used in Cheng \& Castro (2002) may yield a steeper IBL than that by using the three-dimensional cuboid type elements in the current study.

\subsection{Point source dispersion}

The point source dispersion was simulated by a source placed at point ' $\mathrm{S}$ ' in Fig. 1 at $x=14 h$ and $z=-1.5 h$. Although the source size and location were 
similar in the experiment and LES simulations, the source shape was substantially simplified in the LES.

The mean scalar concentration $\bar{C}$ was normalized as follows:

$$
\overline{C^{*}}=\bar{C} \frac{U_{r} L_{r e f}^{2}}{Q}
$$

where the characteristic length $L_{r e f}$ was the building height $h$ and $Q$ was the emission rate. Because LES predictions with inlet-outlet periodic boundary conditions differed from the experimental wind profile and turbulence statistics above $y \sim 3 h$ (Castro et al., 2017), the mean velocity at $y=3 h$ and $x=-2.5 h$ was chosen here as the reference velocity $U_{r}$. Similarly, the scalar variance $\overline{c^{\prime} c^{\prime}}$ was normalized as:

$$
\overline{c^{\prime} c^{* *}}=\left[\frac{\sqrt{\overline{c^{\prime} c^{\prime}}} U_{r} L_{r e f}^{2}}{Q}\right]^{2} .
$$

Coceal et al. (2007) defined the near-field as being within a distance of $2 \sim 3 h$ from the source. Within the near-field the results are likely to be affected by the source shape, size and location, but in the far-field $(>3 h)$ turbulent mixing would be expected to show little memory of the source characteristics and the results are expected to be insensitive to the shape of the source.

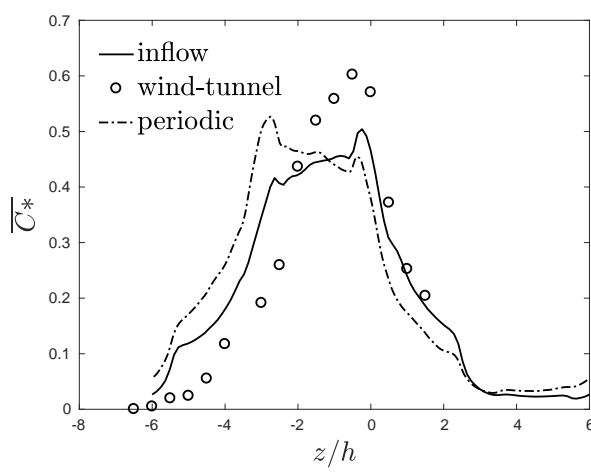

(a)

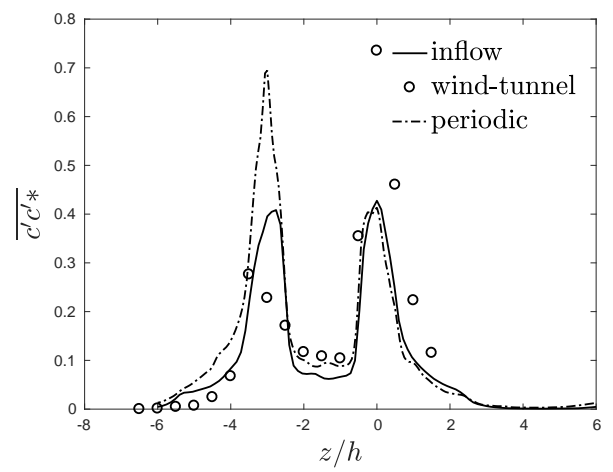

(b)

Figure 8: (a) Inflow, periodic and wind tunnel normalized mean concentration values measured at $x=16 h$ and $y=0.5 h$, resulting from a source at $x=14 h$ and $z=-1.5 h$. (b) Normalized mean scalar variance measured at $x=16 h$ and $y=0.5 h$. 
The IBC LES concentration data were compared against the PBC LES and wind tunnel experiment data reported in Fuka et al. (2017). The first comparison was of data taken in the near-field along a lateral line at $x=16 h$ and $y=0.5 h$. For both sets of LES data the averaging process was long enough (180 flow-passes) to give fully converged results. The results for the dimensionless mean concentration and scalar variance are shown in Fig. 8a and Fig. 8b respectively. The wind tunnel standard error for the mean concentration $\bar{C}$ was $2 \%$.

Both PBC and IBC results for mean concentration in the near-field (Fig. 8a) showed two non-symmetric peaks located in alignment with the corners of the upwind obstacle. Recalling that the source was located in the centre of the long 'street' (position $z=-1.5 h$ ), the IBC highest peak was located on the right side of the source and the PBC highest peak on the left (when looking downwind). In contrast, the experimental data showed a single peak on the right side of the source at $z=0 h$.

The flow around the obstacle downwind of the source determines how the scalar plume divides into the left and/or right channels. In the experiment and the IBC case the plume was found to be mainly transported down the righthand street, rather than the left. The asymmetry in the experimental results was suspected to be due to imperfect alignment of the array and/or a small effective offset in the flow direction. The wind tunnel alignment error is expected to be of the order of $0.25^{\circ}$ (Fuka et al. 2017).

The PBC LES simulation had periodic boundary conditions applied to the lateral sides of the domain, so symmetric results might be expected in the spanwise direction. That the results were found to be asymmetric was perhaps due to strong 3-dimensional anisotropic turbulence leading to non-zero spanwise velocity on the lateral boundaries.

Whether the peaks matched on either the right or left side close to the source was considered to be arbitrary, and of little importance to the results of any farfield analysis. The near-field LES and wind tunnel results were both sensitive to the local flow details at the $0^{\circ}$ wind direction. Except for the peak alignment 


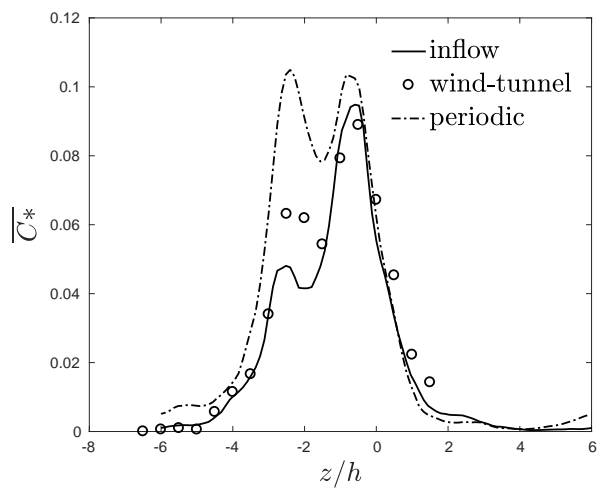

(a) be drawn.

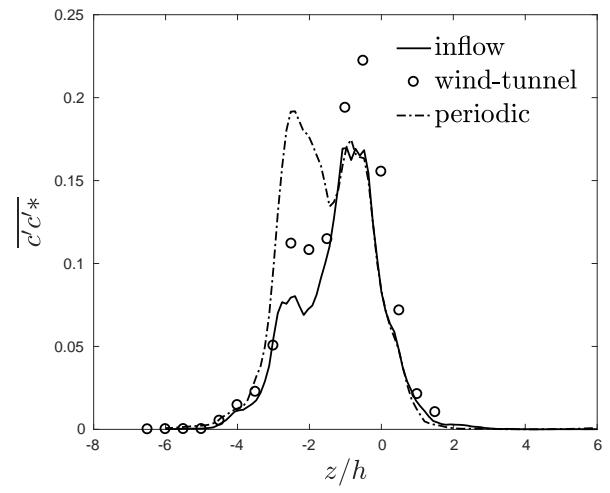

(b)

Figure 9: (a) Inflow, periodic and wind tunnel normalized mean concentration data measured above the canopy at $x=16 h$ and $y=2 h$, for a source at $x=14 h$ and $z=-1.5 h$. (b)

Normalized mean scalar variance measured at $x=16 h$ and $y=2 h$.

discrepancies discussed above, the LES with IBC simulation captured the lateral

Numerical predictions and measurements of the spanwise scalar variance at a height of $y=0.5 h$ and distance $x=16 h$ are compared in Fig. 8b. The experimental results again show a higher peak to the right of the source position, indicating that the plume drifted to the right, whereas the LES with PBC results show a higher peak to the left of the source. The LES with IBC shows two peaks which are almost symmetric about the source position. These results are consistent with those discussed above. As no standard error data are available for the experimental scalar variance measurements no further conclusions can

Fig. $9 \mathrm{a}$ and Fig. $9 \mathrm{~b}$ show a comparison of the spanwise variation in mean concentration and variance data above the canopy at $y=2 h$ and $x=16 h$. In this case some of the wind tunnel sampling stations might approach or cross the edge of the plume. If this was so, fluctuations in concentration and intermittency would make accurate agreement between modelling and experiment difficult to achieve. Fig. 9a also shows that the mean concentration profiles are not in a 
Gaussian shape. Nevertheless, the IBC results were found to be in fair agreement with the wind tunnel measurements, with both the magnitude and the lateral size of the plume being well predicted.

Although the alignment of both the IBC and experimental plumes on the same side is considered to be fortuitous, the higher peak is well captured and the lower peak only slightly underestimated. The double peak of the measured variance was also fairly well predicted by the IBC method. The PBC results were also in fair agreement with the wind tunnel measurements in terms of magnitude and the lateral size of the plume. In contrast to the experimental and IBC LES results, the PBC LES results show almost symmetric double peaks for the mean concentration, although there is more asymmetry in the variance. The IBC results were qualitatively closer to the experimental data than the PBC ones.

From the results above, it appears that based on the array geometry and locations examined, the IBC method leads to a superior prediction of scalar dispersion than the PBC one, in that it captures the asymmetry observed. This is believed to result from the better agreement between measurements of Reynolds stresses and IBC predictions immediately above the canopy $\S 4.1$.

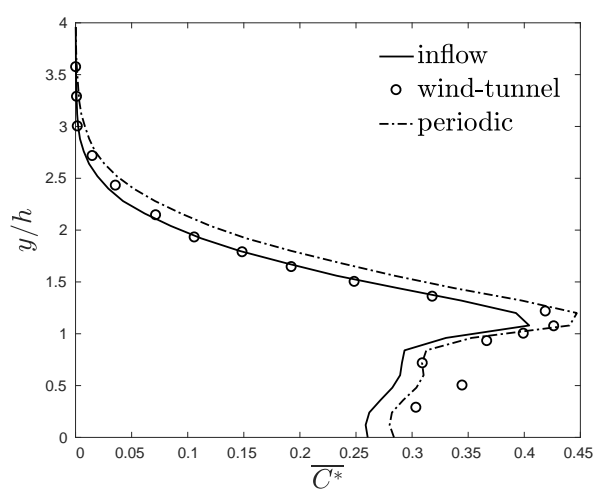

(a)

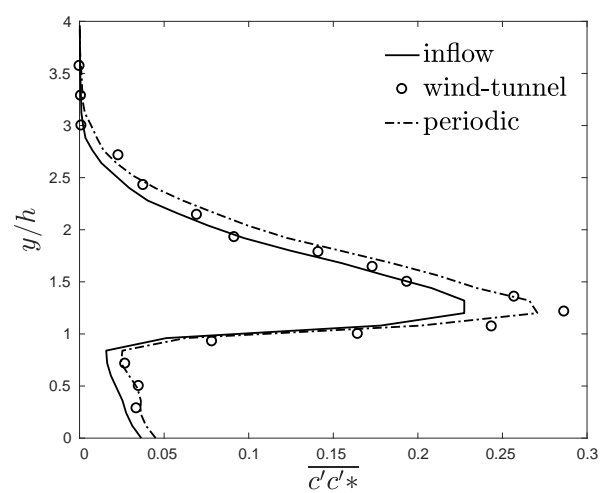

(b)

Figure 10: (a) Inflow, periodic and wind tunnel normalized mean concentration measured at $x=18 h$ and $z=-1.5 h$, resulting from source at $x=14 h$ and $z=-1.5 h$. (b) Normalized mean scalar variance measured at $x=18 h$ and $z=-1.5 h$. 
Further comparisons were made between simulations and measurements of mean concentration and variance in the far-field along a vertical line at $x=$ 18h, $z=-1.5 h$ (Fig. 10a and Fig. 10b). Examination of Fig. 10a shows that both LES IBC and PBC predictions for mean concentration and concentration variance are in fair agreement with the experimental results below $y=1.5 h$. However, the IBC predictions are consistently better than the PBC ones above $y=1.5 h$. Given the differences observed in the near-field, the similarity between these IBC and PBC far-field results confirms that beyond two rows downstream of the source, the effect of the difference in size and shape of the source becomes negligible and one can expect more accurate comparisons.

\subsection{Interface effects on dispersion}

The mechanisms that affect dispersion below and above the urban canopy depend on the position of the source. For example, if the source is placed in the wake of an obstacle in a recirculation zone, the plume is effectively transported upwards either by the mean flow or by the turbulent Reynolds stresses (Fuka et al. 2017; Tomas et al., 2017; Brixey et al., 2009). The dimensionless vertical flux components were defined in Fuka et al. (2017) as follows:

$$
\psi_{a d v}^{v *}=\bar{V} \bar{C} \frac{h^{2}}{Q}
$$

$$
\psi_{t u r b}^{v *}=\overline{v^{\prime} c^{\prime *}}=(\overline{V C}-\bar{V} \bar{C}) \frac{h^{2}}{Q}
$$

where $v^{\prime}$ and $c^{\prime}$ are the vertical velocity fluctuation and the scalar fluctuation respectively and $\bar{V}$ is the mean vertical velocity. It is these fluxes, the advective vertical concentration flux (Eq.13) and the turbulent vertical concentration flux (Eq. 14), that determine the exchange of pollutants between the canopy flow and the boundary layer above.

In the wakes of obstacles the mean vertical velocity and the vertical velocity fluctuation are not negligible, and both flux components contribute to the upwards transport of the scalar. Above the canopy the mean vertical velocity decreases significantly because the flow is predominantly parallel to the array 
canopy, nevertheless, the vertical fluctuation component may still remain significant. This means though that the vertical turbulent concentration flux may contribute more than the advective flux to the upwards transport of the plume above the canopy and close to the IBL interface.

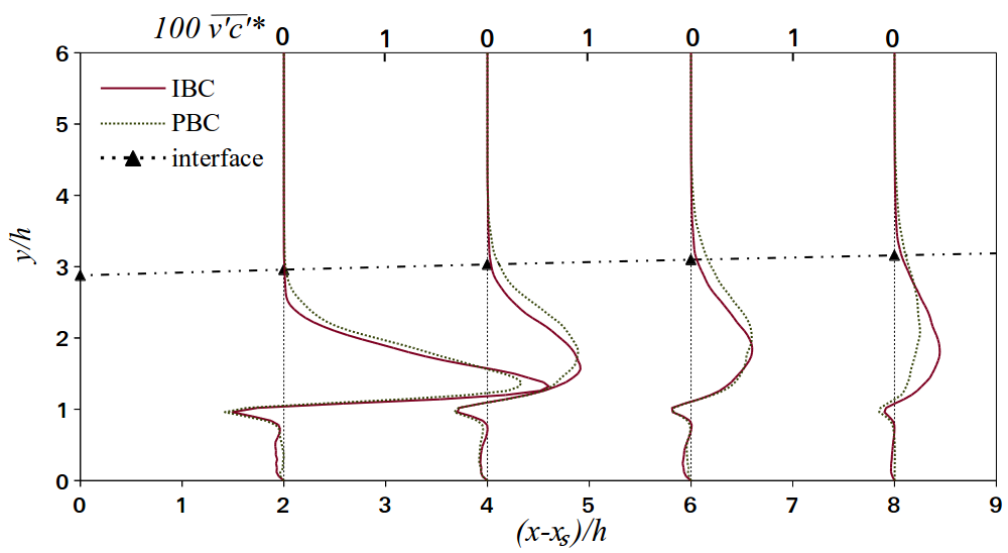

Figure 11: Turbulent vertical flux $\overline{v^{\prime} c^{\prime *}}$ profiles scaled by 100 at four locations downstream of the source position $\left(x_{s}=14 h\right.$ and $z_{s}=-1.5 h$, Fig. 1). The black dashed line shows the IBL interface over the block array.

In Fig. 11, the vertical turbulent flux profile $\overline{v^{\prime} c^{\prime *}}$ (Eq. 14) calculated by using LES IBC and LES PBC predictions is shown at four streamwise locations downstream of the source. The black dashed line represents the interface of the IBL as calculated in $\S 4.2$ using method III. The vertical profiles in Fig. 11 suggest that the edge of the plume grew from the source and matched the interface after 2 rows of cuboids, as further downstream both the interface and the edge of the plume were found to be approximately at the same elevation.

The IBC turbulent flux profiles at all the $\mathrm{x}$-locations analysed decayed sharply when approaching the IBL interface. Looking at the flux profiles at $\left(x-x_{s}\right)=2 h$, a much sharper decay is observed in the IBC profile as the interface is approached than in the PBC one. Similarly, at positions $\left(x-x_{s}\right)=4 h$ and $\left(x-x_{s}\right)=6 h$ the IBC flux profiles decay more rapidly than the PBC profiles when approaching the IBL interface. However, at position $\left(x-x_{s}\right)=8 h$, the IBC vertical flux was observed to be higher than the PBC flux below the 


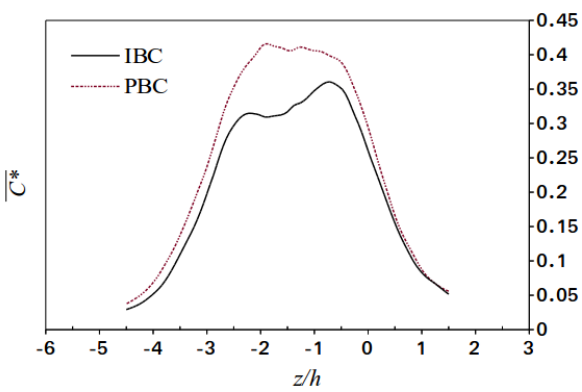

(a) and determines the lateral extent of the plume.

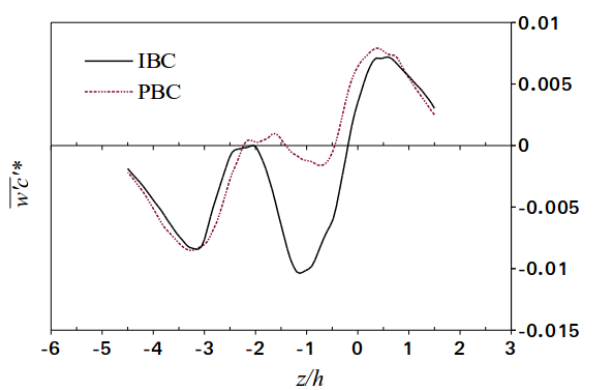

(b)

Figure 12: (a) Spanwise normalized mean concentration predictions at $x=18 h$ and $y=1.5 h$. (b) Spanwise normalized lateral flux predictions at $x=18 h$ and $y=1.5 h$, resulting from a

ground level source at $x=14 h$ and $z=-1.5 h$.

interface, but lower above it. This trend shows that vertical transport of the scalar between the internal and external boundary layers is being constrained by the interface. Moreover, because the IBL interface is defined using method III, the distinct changes in vertical flux profile appear related to similar changes in the vertical Reynolds stress $\overline{v^{\prime} v^{\prime}}$ noted in $\S 4.2$

Dispersion from a ground-level point source is a $3 D$ problem. In order to understand whether vertical constraint by the IBL interface enhanced the lateral spreading of the plume, the lateral turbulent fluxes were also analysed. The turbulent component of the lateral concentration flux was defined as follows:

$$
\psi_{t u r b}^{w *}=\overline{w^{\prime} c^{\prime *}}=(\overline{W C}-\bar{W} \bar{C}) \frac{h^{2}}{Q}
$$

where $w^{\prime}$ and $c^{\prime}$ are the lateral velocity fluctuations and scalar fluctuations respectively, and $\bar{W}$ is the mean lateral velocity. The lateral turbulent flux regulates the diffusion of the plume in positive or negative spanwise directions

Fig. $12 \mathrm{a}$ and $12 \mathrm{~b}$ show the LES IBC and PBC mean concentration and lateral flux predictions at $x=18 h$ (i.e. two rows downstream from the source) and $y=1.5 h$ in the spanwise direction. The spanwise location of the source is $z=-1.5 h$, and the mean concentration is normalized as in Eq. 11. 


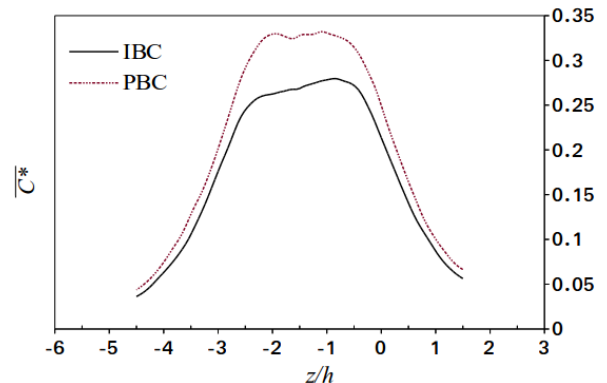

(a) greater than that of the PBC.

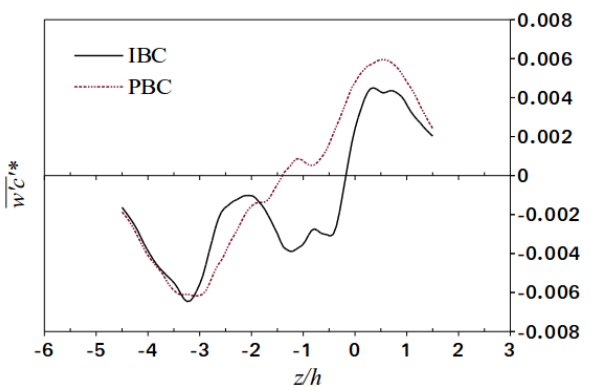

(b)

Figure 13: (a) Normalized mean concentration prediction at $x=20 h$ and $y=1.5 h$. (b) Inflow and periodic normalized lateral flux predictions at $x=20 h$ and $y=1.5 h$, resulting from a source at $x=14 h$ and $z=-1.5 h$.

In common with the results at $x=16 h$ and $y=2 h$ shown in Fig. 9a, the IBC mean concentration in Fig. 12a shows a higher peak located to the right of the source $(z=-0.5 h)$ and a lower peak on the left $(z=-2.5 h)$. Whereas the PBC mean concentration shows one symmetric peak close to the source's position. The double peak in the IBC mean concentration means that there are two zero-crossings of the IBC lateral flux (Fig. 12b). Between $z=-2.5 h$ and $z=-0.5 h$, the magnitude of the IBC lateral flux is much greater than that of the PBC flux. Outside of this range, the fluxes are very close in magnitude.

We fitted the mean concentration profiles to Gaussian distributions, and found that the width of the Gaussian profile for the IBC plume was slightly greater than that of the PBC. This is interesting given the greater lateral flux of the IBC. Fig. 4a shows that the mean streamwise velocity immediately above the canopy in the IBC simulation and the experiment are greater than that of the PBC, which yields a shorter convection time for the plume to develop. This might explain why the plume width above the canopy of the IBC is only slightly

Following the same approach, the mean concentration and the lateral turbulent flux were analysed at $x=20 h$ (three rows downstream) and $y=1.5 h$ over the spanwise direction (Fig. $13 \mathrm{a}$ and $13 \mathrm{~b}$, respectively). The peak PBC mean 
concentration in Fig. 13a is again greater than that of the IBC. The magnitude of IBC lateral flux in Fig. $13 \mathrm{~b}$ is also again greater than the PBC flux near the core of the plume, and the width of the IBC plume was again found to be greater than that of the $\mathrm{PBC}$. These findings are all consistent with those in Figs. $12 \mathrm{a}$ and $12 \mathrm{~b}$.

The mean concentration and lateral turbulent flux profiles in Figures 12 and 13 suggest that the lateral spreading of the IBC plume is greater than that of the PBC plume. This is consistent with the greater lateral Reynolds stresses observed in the IBC profile than in the PBC profile at $y=1.5 h$ shown in Fig. $4 \mathrm{c}$.

In the IBC simulations vertical transport of the scalar between the internal and external boundary layers is constrained by the lower TKE above the interface. The vertical constraint on the vertical Reynolds stress and the enhancement of the lateral Reynolds stress lead to increased lateral spreading of the IBC plume. This results in the lower mean concentrations observed in the IBC simulation than in the PBC one in Fig. 12a and $13 \mathrm{a}$.

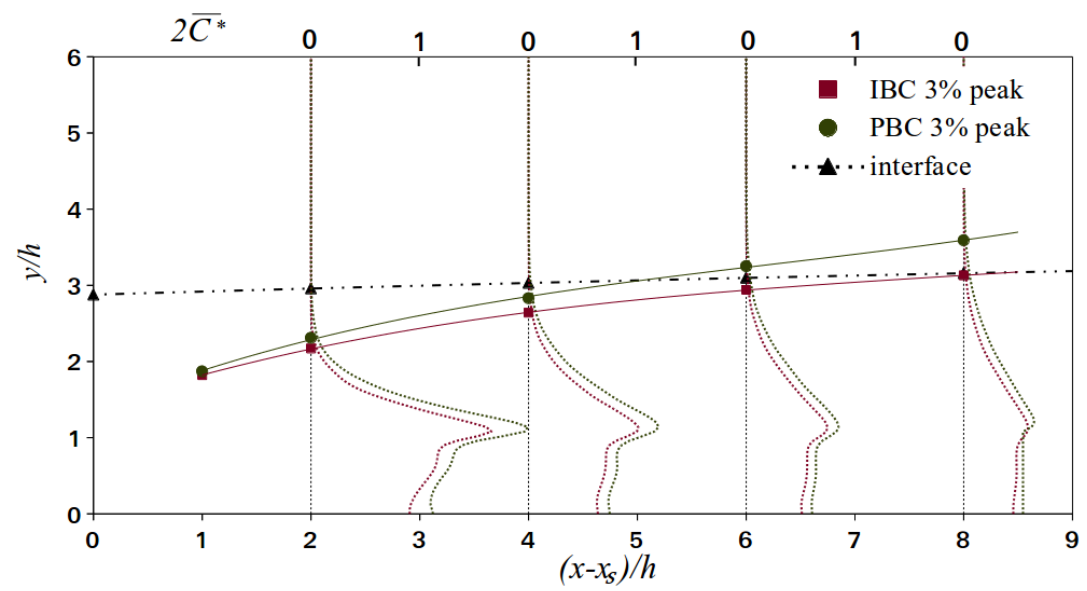

Figure 14: Dimensionless scalar concentration $\bar{C}^{*}$ scaled by 2 in four locations downstream of the source position $\left(x_{s}=14 h, z_{s}=-1.5 h\right.$, Fig. 1 1 . The black dashed line shows the IBL interface. The plume edge is taken as $3 \%$ of the local peak in both LES simulations.

Finally, in order to determine the position of the edge of the plume compared to the IBL interface, vertical profiles of the dimensionless mean concentration 
were taken downstream of the source in several streamwise locations at $z=$ -1.5h (Fig. 14). The results of both PBC and IBC simulations were normalized as in Eq. 11 and compared. The origin of the coordinate system was fixed at the source position.

As far as we are aware, there is no generally accepted definition of the plume's edge. Therefore, the edge of the plume was identified here by considering the $3 \%$ value of the local-peak mean concentration measured at the canopy height. The aim here was to evaluate whether or not the predicted development of the plume when using the IBC method differed from that using the PBC. Hence, the choice of the percentage at which to define the plume edge was not critical. Nevertheless, sensitivity tests made using 1\%, 5\% and $10 \%$ values of the local concentration peak all showed similar plume growth rates.

The development of the plume when using IBC was found to be visibly different from the one by using PBC. This is shown in Fig. 14 in which the plume edge in the IBC simulation appears to asymptote to the IBL interface. Whereas when inlet-outlet PBC are used, the roughness boundary layer grows indefinitely up to the top of the domain which allows the plume to continue to expand vertically. This is a result of the interface between the internal and external boundary layers only existing when the inflow method is applied. Within the simulated LES domain, the plume development appears to be influenced by the IBL interface location which leads to trapping of the scalar in the IBL and greater lateral spreading.

\section{Conclusions and discussion}

LES with prescribed IBC was used to simulate a rural-to-urban transition region where the change in surface roughness generates an IBL at the leading edge of a regular array of cuboids. The LES with IBC was found to provide an accurate simulation of the flow which predicted the TKE to be greater below the interface of the IBL when compared to the TKE obtained from LES with inlet-outlet PBC. 
To our best knowledge the growth rate of the IBL depth has been evaluated for the first time by analysing the vertical Reynolds stress profiles in several streamwise positions. The vertical Reynolds stress method was found to define the IBL interface more clearly than existing methods based on mean streamwise velocity and streamwise Reynolds stress. It was further found that the IBL growth rate derived from the vertical stress method followed the power-law formula with a similar coefficient $a$ as that derived by Cheng \& Castro (2002) in the near roughness transition region $\left(X / z_{02}<1000\right)$, but with a significantly lower exponent $P=0.21$ compared to $P=0.33$ derived by Cheng \& Castro (2002). We speculate this is owing to the difference of characteristics of the roughness elements within a range $X / z_{02}<1000$.

LES predictions of turbulence and dispersion from a ground-level point source were compared against wind tunnel measurements reported in Castro et al. (2017) and Fuka et al. (2017). The impact of the interface between the internal and external boundary layers on dispersion was then analysed by studying vertical and lateral profiles of dimensionless mean concentration and turbulent fluxes downstream of the source. The IBC vertical scalar turbulent flux profiles decayed more rapidly than the PBC profiles when approaching the IBL interface. We speculate that the distinct changes in IBC vertical flux profiles appear related to similar changes in the vertical Reynolds stress. Furthermore, the lateral spreading of the IBC plume was found to be greater than that of the PBC plume, this was found to be correlated with greater values of lateral turbulent Reynolds stress. These features led to the plume's upper edge in the IBC simulation differing distinctly from that obtained by using inlet-outlet PBC. It is concluded that the presence of the IBL constrains vertical spreading, and so leads to trapping of the scalar. The development of the interface between the internal and external boundary layers will also be affected by thermal stratification conditions, and further work should seek to quantify this.

Acknowledgements. VS is grateful to Defense Science and Technology Laboratory and the University of Southampton for the funding of PhD studentship. 
We thank to the EnFlo team at the University of Surrey for providing the wind tunnel data through the DIPLOS project and the appropriate publications. We are grateful to Prof Ian P. Castro, Dr Glyn Thomas and Mr Timothy Foat for helpful comments. The relevant data are available from the University of Southampton database, under the https://doi.org/10.5258/SOTON/D06037.

\section{References}

Antonia, R. A., \& Luxton, R. E. (1972). The response of a turbulent boundary layer to a step change in surface roughness. part 2. rough-to-smooth. Journal of Fluid Mechanics, 53, 737-757. doi:10.1017/S002211207200045X.

Antoniou, N., Montazeri, H., Wigo, H., Neophytou, M., Blocken, B., \& Sandberg, M. (2016). CFD and wind-tunnel analysis of outdoor ventilation in a real compact heterogeneous urban area: Evaluation using air delay. Building and Environment, 126, 355-372. doi:10.1016/j.buildenv.2017.10.013.

Baker, J., Walker, H. L., \& Cai, X. (2004). A study of the dispersion and transport of reactive pollutants in and above street canyonsa large eddy simulation. Atmospheric Environment, 38, 6883 - 6892. doi/https://doi.org/ $10.1016 / \mathrm{j}$. atmosenv.2004.08.051.

Barlow, J. F. (2014). Progress in observing and modelling the urban boundary layer. Urban Climate, 10, Part 2, 216 - 240. doi http://dx.doi.org/10. 1016/j.uclim.2014.03.011. ICUC8: The 8th International Conference on Urban Climate and the 10th Symposium on the Urban Environment.

Barlow, J. F., \& Coceal, O. (2008). A review of urban roughness sublayer turbulence. Technical Report Met Office. URL: http://centaur.reading. ac.uk/38572/,

Bercin, K. M., Xie, Z.-T., \& Turnock, S. R. (2018). Exploration of digital-filter and forward-stepwise synthetic turbulence generators and an improvement for their skewness-kurtosis. Computers \& Fluids, 172, 443 - 466. doi https: //doi.org/10.1016/j.compfluid.2018.03.070. 
Bradley, E. F. (1968). A micrometeorological study of velocity profiles and surface drag in the region modified by a change in surface roughness. Quarterly Journal of the Royal Meteorological Society, 94, 361-379. doi:10.1002/qj. 49709440111.

Brixey, L. A., Heist, D. K., Richmond-Bryant, J., Bowker, G. E., Perry, S. G., \& Wiener, R. W. (2009). The effect of a tall tower on flow and dispersion through a model urban neighborhood part 2. pollutant dispersion. J. Environ. Monit., 11, 2171-2179. doi:10.1039/B907137G

Cao, S., \& Tamura, T. (2007). Effects of roughness blocks on atmospheric boundary layer flow over a two-dimensional low hill with/without sudden roughness change. Journal of Wind Engineering and Industrial Aerodynamics, 95, 679-695. doi:10.1016/j·jweia.2007.01.002.

Castro, I. P., Cheng, H., \& Reynolds, R. (2006). Turbulence over urban-type roughness: Deductions from wind-tunnel measurements. Boundary-Layer Meteorology, 118, 109-131. doi 10.1007/s10546-005-5747-7.

Castro, I. P., Xie, Z. T., Fuka, V., Robins, A. G., Carpentieri, M., Hayden, P., Hertwig, D., \& Coceal, O. (2017). Measurements and computations of flow in an urban street system. Boundary-Layer Meteorology, 162, 207-230. doi:10.1007/s10546-016-0200-7.

Cheng, H., \& Castro, I. P. (2002). Near-wall flow development after a step change in surface roughness. Boundary-Layer Meteorology, 105, 411-432. doi:10.1023/A: 1020355306788 .

Coceal, O., Dobre, A., Thomas, T. G., \& Belcher, S. E. (2007). Structure of turbulent flow over regular arrays of cubical roughness. Journal of Fluid Mechanics, 589, 375-409. doi 10.1017/S002211200700794X

Efros, V., \& Krogstad, P.-A. (2011). Development of a turbulent boundary layer after a step from smooth to rough surface. Experiments in Fluids, 51, 1563-1575. doi $10.1007 /$ s00348-011-1167-2. 
Elliott, W. P. (1958). The growth of the atmospheric internal boundary layer. Eos, Transactions American Geophysical Union, 39, 1048-1054. doi:10.1029/TR039i006p01048

Fuka, V., Xie, Z. T., Castro, I. P., Hayden, P., Carpentieri, M., \& Robins, A. G. (2017). Scalar fluxes near a tall building in an aligned array of rectangular buildings. Boundary-Layer Meteorology, . doi:10.1007/s10546-017-0308-4

Hanna, S., Tehranian, S., Carissimo, B., Macdonald, R., \& Lohner, R. (2002). Comparisons of model simulations with observations of mean flow and turbulence within simple obstacle arrays. Atmospheric Environment, 36, 5067 5079. doi http://dx.doi.org/10.1016/S1352-2310(02)00566-6.

Hanson, R. E., \& Ganapathisubramani, B. (2016). Development of turbulent boundary layers past a step change in wall roughness. Journal of Fluid Mechanics, 795, 494-523. doi:10.1017/jfm.2016.213.

Inagaki, M., Kondoh, T., \& Nagano, Y. (2005). A mixed-time-scale sgs model with fixed model-parameters for practical LES. Journal of Fluids Engineering, 127, 1-13. doi:10.1115/1.1852479.

Jackson, N. A. (1976). The propagation of modified flow downstream of a change in roughness. Quarterly Journal of the Royal Meteorological Society, 102, 924-933. doi:10.1002/qj . 49710243420.

Kanda, I., \& Yamao, Y. (2016). Passive scalar diffusion in and above urbanlike roughness under weakly stable and unstable thermal stratification conditions. Journal of Wind Engineering and Industrial Aerodynamics, 148, 18-33. doi:10.1016/j.jweia.2015.11.002.

Kanda, M., Moriwaki, R., \& Kasamatsu, F. (2004). Large-eddy simulation of turbulent organized structures within and above explicitly resolved cube arrays. Boundary-Layer Meteorology, 112, 343-368. doi 10.1023/B:BOUN. $0000027909.40439 .7 \mathrm{C}$ 
King, M., Gough, H., Halios, C., Barlow, J., Robertson, A., Hoxey, R., \& Noakes, C. (2017). Investigating the influence of neighbouring structures on natural ventilation potential of a full-scale cubical building using timedependent CFD. Journal of Wind Engineering and Industrial Aerodynamics, 169, 265-279. doi:10.1016/j.jweia.2017.07.020.

Michioka, T., Sato, A., Takimoto, H., \& Kanda, M. (2011). Large-eddy simulation for the mechanism of pollutant removal from a two-dimensional street canyon. Boundary-Layer Meteorology, 138, 195-213. doi 10.1007/ s10546-010-9556-2.

Pendergrass, W., \& Arya, S. (1984). Dispersion in neutral boundary layer over a step change in surface roughnessi. mean flow and turbulence structure.

Atmospheric Environment (1967), 18, 1267 - 1279. doi/https://doi.org/ 10.1016/0004-6981(84)90037-4.

Schlichting, H., \& Kestin, J. (1979). Boundary-layer theory. (7th ed.). New York : McGraw-Hill. URL: http://trove.nla.gov.au/work/4900562.

Schofield, W. H. (1975). Measurements in adverse-pressure-gradient turbulent boundary layers with a step change in surface roughness. Journal of Fluid Mechanics, 70, 573-593. doi:10.1017/S0022112075002200.

Smits, A. J., \& Wood, D. H. (1985). The response of turbulent boundary layers to sudden perturbations. Annual Review of Fluid Mechanics, 17, 321-358. doi:10.1146/annurev.fl.17.010185.001541.

Tolias, I., Koutsourakis, N., Hertwig, D., Efthimiou, G., Venetsanos, A. G., \& J.G., B. (2018). Large eddy simulation study on the structure of turbulent flow in a complex city. Journal of Wind Engineering and Industrial Aerodynamics, 177, 101-116. doi:10.1016/j.jweia.2018.03.017.

Tomas, J. M., Eisma, H. E., Pourquie, M. J. B. M., Elsinga, G. E., Jonker, H. J. J., \& Westerweel, J. (2017). Pollutant dispersion in boundary layers exposed to rural-to-urban transitions: Varying the spanwise length scale of 
the roughness. Boundary-Layer Meteorology, 163, 225-251. doi 10.1007/ s10546-016-0226-x

Townsend, A. A. (1965). Self-preserving flow inside a turbulent boundary layer. Journal of Fluid Mechanics, 22, 773-797. doi:10.1017/S0022112065001143

Vasaturo, R., Kalkman, I., Blocken, B., \& van Wesemael P.J.V. (2018). Large eddy simulation of the neutral atmospheric boundary layer: performance evaluation of three inflow methods for terrains with different roughness. Journal of Wind Engineering and Industrial Aerodynamics, 173, 241-261. doi:10.1016/j.jweia.2017.11.025.

Wood, D. H. (1982). Internal boundary layer growth following a step change in 口 surface roughness. Boundary-Layer Meteorology, 22, 241-244. doi 10.1007/ BF00118257.

Xie, Z. T., \& Castro, I. P. (2006). LES and RANS for turbulent flow over arrays of wall-mounted obstacles. Flow, Turbulence and Combustion, 76, 291-312. doi $10.1007 / \mathrm{s} 10494-006-9018-6$.

Xie, Z. T., \& Castro, I. P. (2008). Efficient generation of inflow conditions for large eddy simulation of street-scale flows. Flow, Turbulence and Combustion, 81, 449-470. doi 10.1007/s10494-008-9151-5. 